\title{
Probabilistic Brain Atlas Construction: Thin-Plate Spline Warping via Maximization of Mutual Information
}

\author{
C.R. Meyer, J.L. Boes, B. Kim, and P.H. Bland \\ Department of Radiology \\ University of Michigan, 3307 Kresge III Research, Ann Arbor, MI USA 48109-0553 \\ cmeyer@umich.edu
}

\begin{abstract}
The concepts of a probabilistic atlas are well known. The dispersions of the resulting atlas' spatial probability distributions depend not only on the intrinsic variation of structures between subjects, but also on the ability of the intersubject mapping method to compensate for gross spatial variations. We demonstrate an automatic method of registering patients to an atlas by maximization the mutual information between the atlas and the patient's gray scale data set. The global thin-plate spline (TPS) transformation for mapping each subject is computed by automatically optimizing the loci of 40 control points distributed within the atlas. The use of 40 control points, i.e. $3 * 40=120$ degrees of freedom (DOF), is a compromise between viscous flow methods with huge DOF, and the 12 DOF affine mapping. We quantitatively compare the results between using a full affine transformation versus the MIdriven 40 control point thin-plate spline for the mean and standard deviation volume data sets computed over the gray scale volumes of 7 patients.
\end{abstract}

\section{Introduction}

For several years many noted groups have been both building probabilistic atlases of the human head as well as developing methods to further improve the classification specificity of such atlases. Evans, et al, developed early automatic techniques using affine transformations [1]. Since then the same group has developed a scale space incremental deformation method called ANIMAL and have pursued additional constraints [2] while applying such atlases to the detection of abnormalities such as multiple sclerosis [3] and epilepsy [4]. Thirion, et al, have pursued the registration of "crest lines" in the creation of atlases [5-9]. Christensen has demonstrated the ability to build atlases using viscous fluid flow models [10, 11], and Thompson has added additional free boundary surface constraints to the viscous flow deformation technique [12]. Our objective was to apply the global objective criterion of maximizing mutual information in developing an automatic method where the degrees of freedom (DOF) were sufficiently small so as to prevent unjustified local deformations, e.g. as in creating a tumor to match the patient's data using a tumorless model, but still had sufficient DOF and robustness to more accurately deform to fit a 
wider variation in patient geometries. The use of mutual information is attractive because the atlas matching technique is independent of modality or acquisition parameters as experienced in MRI. This middle ground approach yields a solution that requires only the use of current generation desktop workstations for reasonable case compute times.

In this paper we report on the quantitative and visual differences obtained by mapping 7 patients of significantly different brain anatomy into the geometry of the atlas using both the full affine, 12 DOF transform, and a 120 DOF thin-plate spline transform under automatic control.

\section{Methods}

The atlas was obtained from http://www.bic.mni.mcgill.ca/brainweb/), the web site of the McConnell Brain Imaging Centre, Montreal Neurological Institute, McGill University. The atlas was constructed under the direction of Alan Evans with funding from the International Consortium for Brain Mapping (ICBM). The ICBM atlas is a $1 \mathrm{~mm}$ cubic voxel MRI head scan that has been manually segmented. We modified our version of the atlas such that region 1 consists of cerebrospinal fluid, region 2 is gray matter, region 3 is white matter, and region 0 fills the remainder of the volume.

All patients were imaged using a GE 1.5 T MRI using a 3D T1-weighted, gradient recalled echo sequence with inversion recovery preparation which produces excellent gray/white matter contrast and B1-field uniformity (no subsequent B1-field correction was applied). For the registration method we used our "MIAMI Fuse" software package where the acronym stands for mutual information for automatic multimodality image fusion[13]. The subsampled ICBM atlas was chosen as the reference target into which to map the patient data volume. The atlas was decimated by using every third voxel in $\mathrm{x}$ and $\mathrm{y}$, and every other voxel in $\mathrm{z}$. Forty control point locations were spatially distributed in the atlas to yield relatively uniform distances between control points and provide local shape control near structures of large variability such as the ventral and dorsal horns of the ventricles.

To begin the mapping of the patient into the atlas, the user begins the process by identifying 4 control points in the patient's data volume that are approximately homologous to the first 4 control points in the atlas' set of 40 . The method first optimizes a 7 degree-of-freedom (DOF) affine geometric fit (rotation, isotropic scaling, and translation computed from 4 control points) between the patient data and the ICBM model until the increase in mutual information between repeated optimization cycles is less than .0001 bit. The method continues by fitting a 12 DOF full affine model also using only 4 control points, followed a 120 DOF fit using 40 control points for a thin plate spline (TPS) warping model. At each DOF the same stopping criterion is used before proceeding to the next DOF level. The optimal solution at one DOF level is used as the initial starting condition for the next level by mapping the desired number of control points from the atlas into the patient data set using the previous, best solution. In this manner the patient MRI is first mapped onto the ICBM model using large scale, global controls while more local controls are applied last. 


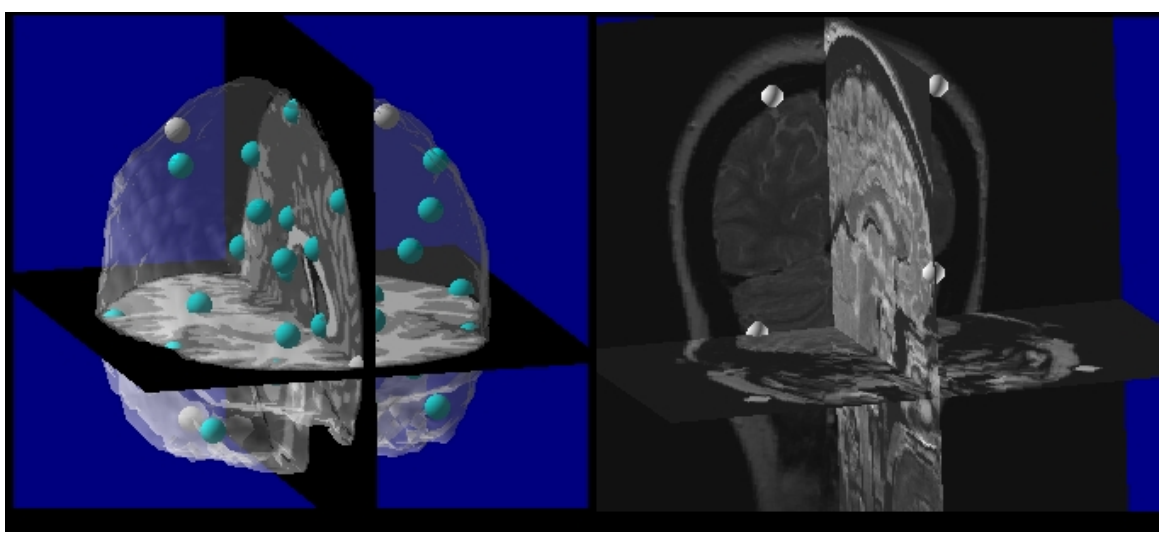

Fig. 1a: View of ICBM atlas with 40 Fig. 1b: View of typical patient data control points distributed over set with 4 approximate homologous volume. The first 4 control points are control points placed in volume to depicted in white. roughly match the positions of the white markers in atlas

The optimizer used (to minimize the negative of mutual information) was the Nelder-Mead Simplex method commonly referred to as amoeba [14]. While not optimal in the number of iterations required to reach the solution vector in noiseless function optimization problems, the simplex method behaves very much like simulated annealing with its own computed cooling schedule, and is very robust against being trapped by local minima in noisy cost functions. The n-vector to be optimized consists of the coordinate positions of the control points in the patient data set where $n$ equals three times the number of control points. Each optimization cycle is terminated when the requested movement of each of the control points in the patient volume set in any of the 3 cardinal coordinate directions is less than $0.1 \mathrm{~mm}$.

After registration with the atlas, all patient data sets were gray scale amplitude normalized from the same white matter volume of interest. Next the mean and standard deviation gray scale volumes were computed for each of the two registration methods, full affine and 120 DOF TPS. Since these data sets were registered to a labeled atlas, we are able to easily dissect the distributions of gray scale values of these mappings for the specific labels, i.e. cerebrospinal fluid, gray matter, and white matter, and the overall combination. 


\section{Results}

The following table reports the quantitative results for the combination of patients for the two different mapping methods.

\begin{tabular}{|c|c|c|c|c|c|c|c|c|}
\hline \multicolumn{9}{|c|}{ Metrics on MRI Gray Scale Distributions Across Patients } \\
\hline & \multicolumn{4}{|c|}{40 Point TPS Warping } & \multicolumn{4}{|c|}{ For Full Affine Mapping } \\
\hline $\begin{array}{l}\text { Selected } \\
\text { Volumes }\end{array}$ & CSF & Gray & White & $\begin{array}{l}\text { Com- } \\
\text { bined }\end{array}$ & CSF & Gray & White & $\begin{array}{l}\text { Com- } \\
\text { bined }\end{array}$ \\
\hline & \multicolumn{8}{|c|}{ Computed Standard Deviation Volume } \\
\hline mean & 10.95 & 10.15 & 8.47 & 9.73 & 12.13 & 10.67 & 9.4 & 10.52 \\
\hline $2.5 \%$ tile & 3 & 3 & 2 & 3 & 3 & 3 & 2 & 2 \\
\hline $97.5 \%$ tile & 30 & 20 & 20 & 20 & 32 & 21 & 23 & 24 \\
\hline \multirow[b]{2}{*}{ mean } & \multicolumn{8}{|c|}{ Computed Coefficient of Variation Volume } \\
\hline & 0.47 & 0.30 & 0.18 & 0.29 & 0.48 & 0.31 & 0.21 & 0.31 \\
\hline $2.5 \%$ tile & 0.15 & 0.05 & 0.02 & 0.02 & 0.16 & 0.06 & 0.02 & 0.02 \\
\hline $97.5 \%$ tile & 0.95 & 0.66 & 0.52 & 0.75 & 1.00 & 0.72 & 0.60 & 0.80 \\
\hline
\end{tabular}

Table 1.

More local effects can be observed by viewing both the mean and standard deviation volumes. Figures $2 \mathrm{a}$ and $2 \mathrm{~b}$ show the same axial slice through the average volumes obtained by the two methods. Although the global objective function used for the mapping, i.e. mutual information, was computed only over the brain tissues of the atlas, Fig. 2 shows the average including extradural tissues so the reader can view the dural edges without clipping. Figures $3 \mathrm{a}$ and $3 \mathrm{~b}$ show the same sagittal slice through the calculated standard deviation volume. The grayscale window-level was chosen such that white represents a standard deviation of 70 and black represents 0 .

Additionally the graph in Fig. 4 depicts the improvement in the objective function, mutual information, and the average total number of optimization iterations required as the number of control points is varied between 4 and 40 points. The compute time using 40 control points was on the order of 11.5 hours on a $433 \mathrm{MHz}$ Digital Equipment Corporation (DEC) single processor, Personal Alpha workstation running DEC UNIX 4.0. 


\section{Discussion}

The first observation we make is that, similar to Collins [2], the global quantitative metrics understate the marked improvement that can be visualized in the images. Thus even though some small improvement in variance averaged over the atlas volume can be discerned between the two methods in Table 1, the automatic TPS

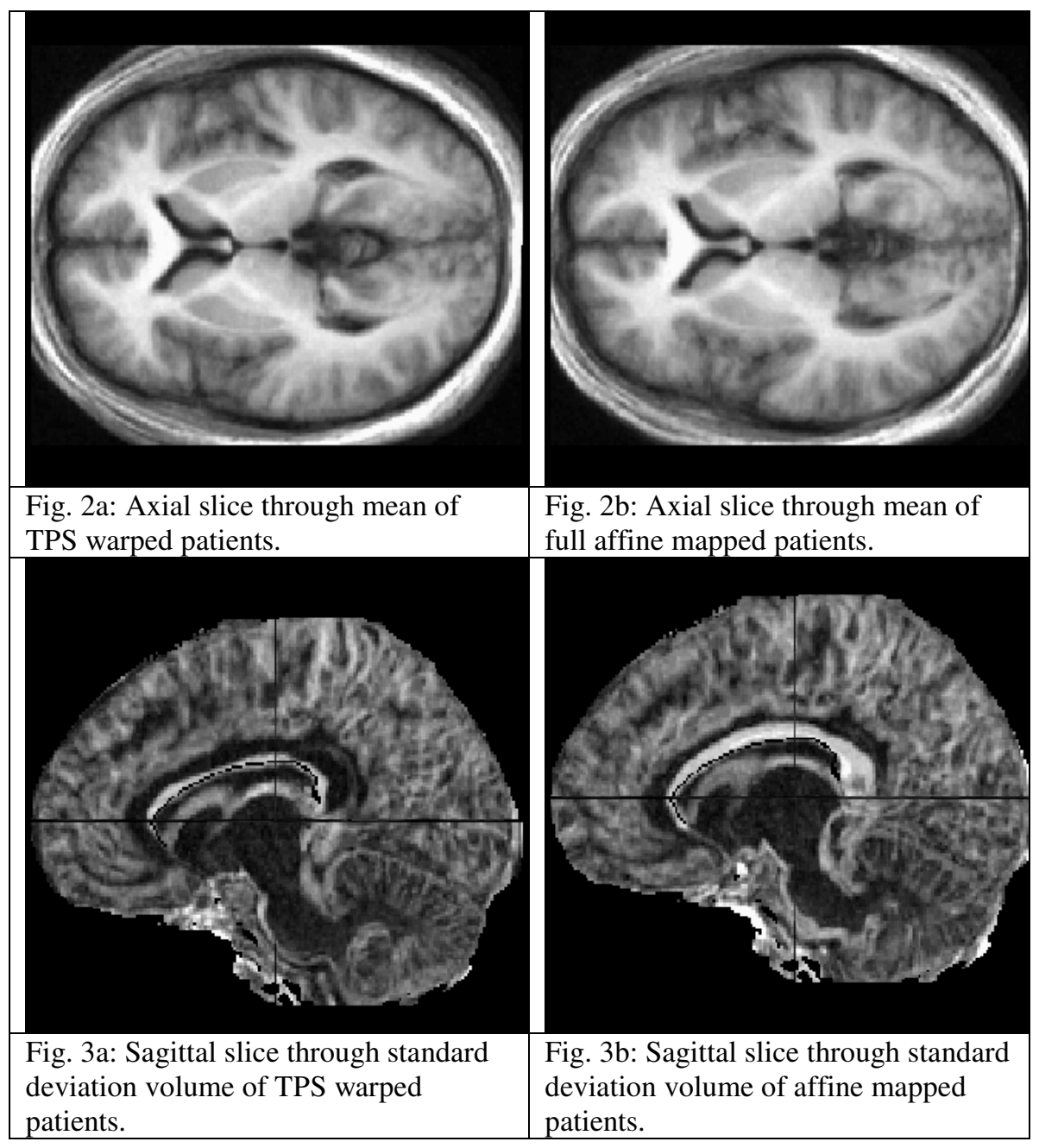

warping results in an improved clarity of structures whether midbrain, or more peripheral. In Fig. 2a note the improved clarity of the putamen, globus pallidus, and caudate nuclei, as well as the regions near the vermis of the cerebellum, posterior ventricles, and lateral sulcus. In Fig. 3 the most striking difference is in the local standard deviation associated with the registration of the corpus callosum and ventricle; note the much thicker region of high standard deviation surrounding these 
midbrain regions as well as the edges of the thalamus and pons. Registration near the dura of the occipital cortex is noticeably improved as well.

From the following graph we observe that there are significant, easy gains in registration accuracy associated with small increases in degrees of freedom (DOF) beyond 12, i.e. 4 control points. Additionally we observe that the improvement in MI with increasing numbers of control points does not appear to be asymptotically approaching an upper threshold at 40 control points, which suggests that we can significantly increase, e.g. double, the number of control points and improve the resulting fit even further. Since the increase in iterations appears to be nearly linear with number of control points, the computational cost of using more control points should not be onerous.

In summary we have demonstrated an implementation of a method for automatically computing a human brain atlas using the mutual information (MI) maximization criterion where the variable degrees of freedom are specified by the number of control points used. The use of MI makes the registration of any MRI acquisition sequence trivial, and the use of limited, i.e. 120, degrees of freedom helps insure the unique mapping of similar structures across patients. The process is robust and typically requires no user intervention or repeated computational runs due to divergent registrations.

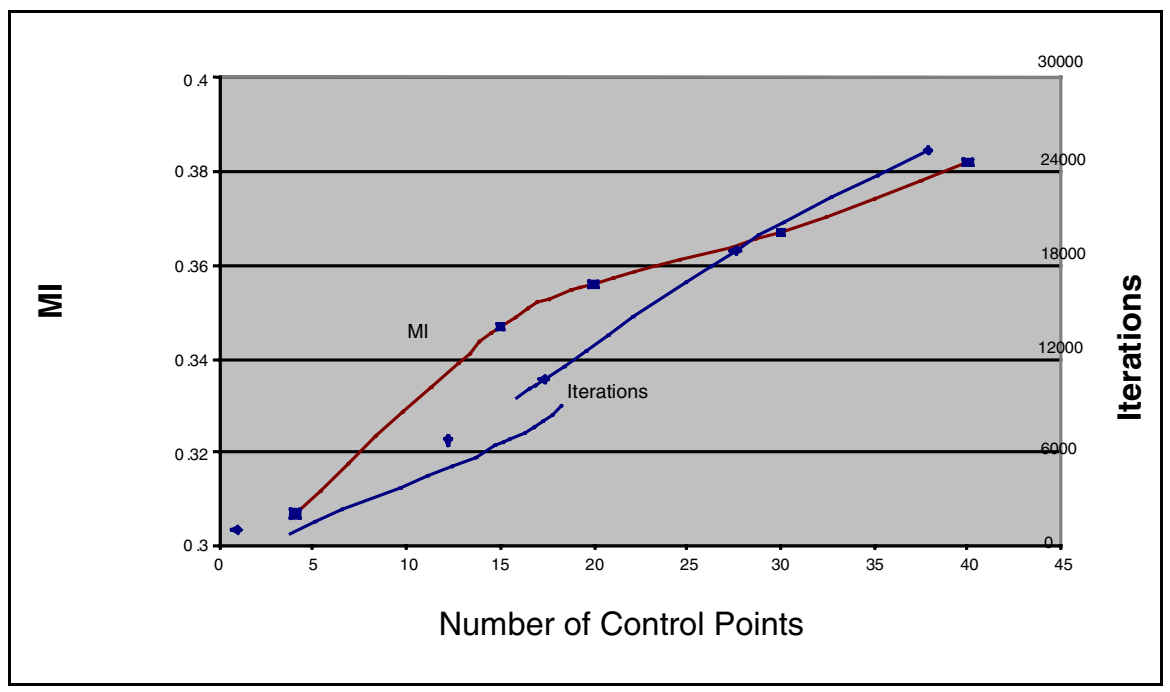

\section{References}

[1] D. L. Collins, T. M. Peters, and A. C. Evans, "An automated 3D non-linear image deformation procedure for determination of gross morphometric variability in human brain,", Rochester, MN, 1994.

[2] D. L. Collins, G. L. Goualher, R. Verugopal, A. Caramanos, A. C. Evans, and C. Barillot, "Cortical constraints for nonlinear cortical registration," presented at Visualization in Biomedical Computing, Hamburg, Germany, 1996. 
[3] A. Zijdenbos, A. Evans, F. Riahi, J. Sled, J. Chui, and V. Kollokian, "Automatic quantification of multiple sclerosis lesion volume using stereotaxic space," presented at Visualization in Biomedical Computing, Hamburg, Germany, 1996.

[4] J. W. Lee, F. Dubeau, A. Bernasconi, D. MacDonald, A. Evans, and D. C. Reutens, "Morphometric analysis of the temporal lobe in temporal lobe epilepsy," Epilepsia, vol. 39, pp. 727-736, 1998.

[5] X. Pennec and J. Thirion, "A framework for uncertainty and validation of 3D registration methods based on points and frames," Internat J Computer Vision, vol. 25, pp. 203-229, 1997.

[6] G. Subsol, J.-P. Thirion, and N. Ayache, "Application of an automatically built 3D morphometric brain atlas: study of cerebral ventricle shape," presented at Visualization in Biomedical Computing, Hamburg, Germany, 1996.

[7] J. Thirion, "Image matching as a diffusion process: an analogy with Maxwell's demons," Medical Image Analysis, vol. 2, pp. 243-260, 1998.

[8] J.-P. Thirion, "Non-rigid matching using demons," presented at International Conference on Computer Vision and Pattern Recoginition (CVPR'96), 1996.

[9] J. Thirion, "The extremal mesh and the understanding of 3D Surfaces,", Seattle, 1994.

[10] G. E. Christensen, R. D. Rabbitt, and M. I. Miller, "Deformable templates using large deformation kinetics," IEEE Image Proc, vol. 5, pp. 1435-1447, 1996.

[11] M. I. Miller, G. E. Christensen, Y. Amit, and U. Grenander, "Mathematical textbook of deformable neuroanatomies.," Proc. Natl. Acad. Sci. USA, vol. 90, pp. 11944-8, 1993.

[12] P. Thompson and A. W. Toga, "A surface-based technique for warping three dimensional images of the brain," IEEE TMI, vol. 15, pp. 402-417, 1996.

[13] C. R. Meyer, J. L. Boes, B. Kim, P. Bland, K. R. Zasadny, P. V. Kison, K. Koral, K. A. Frey, and R. L. Wahl, "Demonstration of accuracy and clinical versatility of mutual information for automatic multimodality image fusion using affine and thin plate spline warped geometric deformations," Medical Image Analysis, vol. 3, pp. 195-206, 1997.

[14] W. H. Press, B. P. Flannery, S. A. Teukolsky, and W. T. Vetterling, Numerical Recipes in C: The Art of Scientific Computing. Cambridge: Cambridge University Press, 1988. 